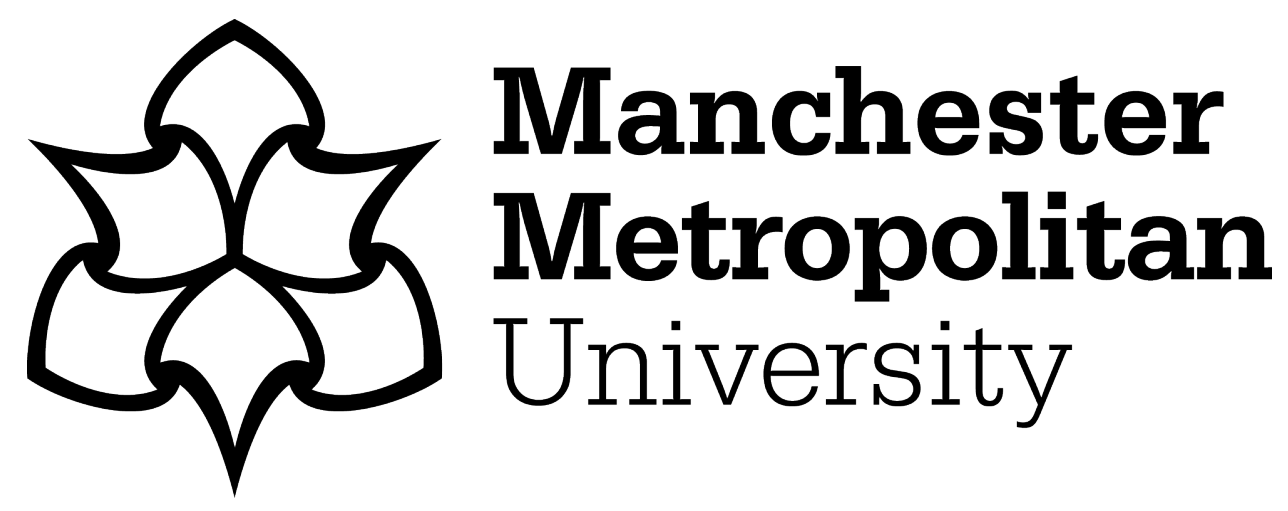

Littlewood, C ORCID logoORCID: https://orcid.org/0000-0002-7703-727X, Bateman, M, Connor, C, Gibson, J, Horsley, I, Jaggi, A, Jones, V, Meakins, A and Scott, M (2019) Physiotherapists' recommendations for examination and treatment of rotator cuff related shoulder pain: A consensus exercise. Physiotherapy Practice and Research, 40 (2). pp. 87-94. ISSN 2213-0683

Downloaded from: https://e-space.mmu.ac.uk/625251/

Version: Accepted Version

Publisher: IOS Press

DOI: https://doi.org/10.3233/PPR-190129

Please cite the published version 


\section{Physiotherapists Recommendations for Examination and Treatment of Rotator Cuff Related Shoulder Pain: A consensus exercise}

Chris Littlewood $\mathrm{PhD}^{1}$, Marcus Bateman $\mathrm{MSc}^{2}$, Clare Connor $\mathrm{MSc}^{3}$, Jo Gibson MSc${ }^{4}$, lan Horsley $\mathrm{PhD}^{5}$, Anju Jaggi $\mathrm{BSc}^{6}$, Val Jones $\mathrm{MSc}^{7}$, Adam Meakins $\mathrm{BSc}^{8}$, Martin Scott $\mathrm{MSc}^{9}$.

${ }^{1}$ Arthritis Research UK Primary Care Centre, Research Institute for Primary Care and Health Sciences and Keele Clinical Trials Unit, Keele University, Staffordshire, UK. c.littlewood@keele.ac.uk

${ }^{2}$ Derby Shoulder Unit, University Hospitals Derby \& Burton NHS Foundation Trust, Derby, UK. marcus.bateman@nhs.net

${ }^{3}$ Aneurin Bevan University Health Board. clareconnor71@hotmail.com

${ }^{4}$ Liverpool Upper Limb Unit, Royal Liverpool University Hospital, Liverpool, UK.

jo.gibson@rlbuht.nhs.uk

${ }^{5}$ English Institute of Sport, Manchester, UK. ian@back-in-action.co.uk

${ }^{6}$ Royal National Orthopaedic Hospital, Middlesex, UK. anjujaggi@ hotmail.com

${ }^{7}$ Sheffield Shoulder and Elbow Unit, Sheffield Teaching Hospital NHS Trust, Sheffield, UK. valerie.jones@sth.nhs.uk

${ }^{8}$ West Herts Shoulder Service, West Hertfordshire Hospital Trust, St Albans Herts, Spire Healthcare, Spire Bushey Hospital, Hertfordshire, UK. a.meakins@nhs.net

${ }^{9}$ Nottingham Shoulder \& Elbow Unit, Nottingham University Hospitals NHS Trust, Nottingham, UK martin.scott@nuh.nhs.uk

Word count $=3662$ 


\section{Abstract}

Background: Disorders associated with the rotator cuff are regarded as the most common shoulder pain presentation. The range of diagnostic terms used to explain this problem reflect uncertainty in relation to causative mechanisms, diagnosis, prognosis, and the most effective treatments. The aim of this consensus exercise was to facilitate a shared understanding as a means of reducing mixed messages, informing clinical practice and providing a foundation for future research.

Methods: Ten physiotherapists with clinical and academic expertise in shoulder pain participated in an online and face-to-face consensus exercise.

Results: This consensus exercise suggests specific factors in the history and physical examination that might raise the index of suspicion of Rotator Cuff Related Shoulder Pain. The suggestions for non-surgical management include a minimal number of exercises prescribed to challenge the functional deficit of the patient over a minimum 12-week period. Apart from aiding exclusion of red flag pathology, imaging is not regarded as useful unless the patient does not respond as expected. Steroid injections wouldn't be considered a first-line intervention unless pain was severe and preventing engagement with exercise.

Conclusion: This consensus exercise provides a benchmark for clinical reflection while highlighting areas of uncertainty that still exist and require further research.

Key words: rotator cuff; diagnosis; exercise; physiotherapy 


\section{Introduction}

Many patients consult healthcare professionals complaining of shoulder pain, with disorders associated with the rotator cuff (subacromial impingement, subacromial pain, rotator cuff related shoulder pain (RCRSP) and other synonyms) widely regarded as the most common presentation $(1,2)$. The wide range of diagnostic or descriptive terms used to explain this common clinical presentation reflect uncertainty in relation to understanding of the pathology, diagnosis, prognosis, and the most effective treatment options (1,3-5). A range of diagnostic tests, including imaging, and a range of different treatment options are available including exercise programmes, manual therapy, corticosteroid injections and surgery (6). Based on recent randomised controlled trials and systematic reviews, where clinical intervention is required, exercise is recommended as the first-line intervention of choice with comparable outcomes but at a lower cost and with fewer associated risks than surgical management. $(7,8)$. Despite this, there is little research evidence to inform what constitutes an effective exercise programme in terms of the type of exercise, the number of sets, repetitions, acceptable pain levels, duration and setting $(9,10)$. A previous systematic review has suggested that inclusion of some level of resistance seems to matter although the optimal level is unclear; the optimal number of repetitions is also unclear but higher repetitions might confer superior outcomes; higher number of sets of exercise are preferable to lower number of sets but the optimal frequency is unknown; most programmes should demonstrate clinically significant outcomes by 12 weeks (9). So, although there is some guidance available, there still remains a number of significant unknowns (9). This is important because it might mean that suboptimal exercise prescription leads to patients moving on to more costly, invasive and risky interventions, including corticosteroid injections and surgery, which are of questionable effectiveness 
$(8,11,12)$. This concern is justified when it is recognised that the practice of physiotherapists across the UK with regards to exercise prescription is highly variable $(6,13)$.

In 2012 a group of international physiotherapists reported their consensus on physiotherapy for shoulder pain (14). This guidance recommended: active exercise prescribed in relation to the clinical assessment and not the structural pathology; mild to moderate pain $(<4 / 10$ on VAS) secondary to the exercise was acceptable but must subside within 12 hours; the quality of the performance of exercise was crucial and exercise should be performed with optimal scapular positioning and control without abnormal compensatory trunk movement; exercises should be simple and slow and unloaded to begin with, proceeding gradually to loaded and faster exercises; the number of exercises should be limited to a maximum of four, and dose and progressions should be individualised (14). Aspects of this consensus reflect the emerging evidence, however in view of the limited progress in understanding the most appropriate terminology, approach to diagnosis, understanding of prognosis, and optimal exercise approach and continued conflicting messages within the literature, the purpose of this paper was to undertake an updated consensus process. The aim was to enable a shared understanding of this common shoulder pain presentation as a means of reducing mixed messages, informing physiotherapy practice and providing a basis on which future research could be developed.

\section{Methods}

In December 2017 the lead author, a physiotherapist, approached nine further physiotherapists recognised for their clinical and academic expertise in the management of shoulder pain. Of the nine approached, eight accepted and formed the consensus group. The physiotherapist who declined did not offer reasons for not participating. 
Subsequent to this, the lead author circulated electronically a list of questions broadly based on approaches taken by other consensus groups (15) and invited commentary from the group. This resulted in a refined list of questions that would be used as the basis for this consensus process (table 1).

\begin{tabular}{|c|c|}
\hline & Question \\
\hline 1 & $\begin{array}{l}\text { What key information is required from the history to raise the index of suspicion of this } \\
\text { classification or diagnosis over other shoulder pain presentations? }\end{array}$ \\
\hline 2 & $\begin{array}{l}\text { Do you consider age, gender and occupation as relevant factors within your diagnostic } \\
\text { reasoning with regard to 'rotator cuff syndrome'? }\end{array}$ \\
\hline 3 & $\begin{array}{l}\text { What key information is required from the physical examination to confirm this classification } \\
\text { or diagnosis? }\end{array}$ \\
\hline 4 & What is the role of imaging in confirming this classification or diagnosis? \\
\hline 5 & What is the preferred terminology for this shoulder pain classification? \\
\hline 6 & In summary, what is 'rotator cuff syndrome'? \\
\hline 7 & How do you explain 'rotator cuff syndrome' to your patients? \\
\hline 8 & What is/ are the preferred methods of treatment? \\
\hline 9 & $\begin{array}{l}\text { Do you consider the role of lifestyle, e.g. sedentary behaviour, and metabolic factors, e.g. } \\
\text { obesity when assessing and treating patients with this pain presentation? }\end{array}$ \\
\hline 10 & $\begin{array}{l}\text { If exercise is prescribed, what are the preferred parameters guiding this prescription? } \\
\text { - } \quad \text { Type of exercise and the factors that inform this } \\
\text { - } \quad \text { Number of sets and repetitions and the factors that inform this } \\
\text { - } \text { Frequency of exercise and the factors that inform this } \\
\text { - } \quad \text { Number of exercises and the factors that inform this } \\
\text { - Intensity/ effort of exercise and the factors that inform this } \\
\text { - Painful versus painless and the factors that inform this } \\
\text { - } \quad \text { Duration of exercise }\end{array}$ \\
\hline 11 & Which factors or characteristics do you see as important indicators of prognosis? \\
\hline 12 & What is the expected prognosis of 'rotator cuff syndrome'? \\
\hline
\end{tabular}

Table 1 List of questions on which the consensus exercise was based 
Following agreement, questions were recirculated to the group, and comments were requested. At the end of this stage, the lead author summarised responses. For example, where two comments had similar purpose and meaning they were amalgamated, without any attempt to interpret the initial responses. After this the group were asked to confirm that the summarised document was a fair summary of their inputs.

The group met face-to-face at the annual British Elbow \& Shoulder Society meeting in Glasgow in June 2018 with a view to generating a consensus statement. Each of the comments, in turn, was presented to the group who were asked to vote whether they agreed with the comment or not. A majority response, i.e. $\geq 5 / 9$, meant that the comment was carried forward and included in the narrative summary.

It was agreed that although a comment carried through to the narrative summary was reflective of the majority of the group, it does not necessarily reflect the view of all individuals all of the time. 


\section{Results}

The results are summarised in table 2 with a full narrative description below.

\begin{tabular}{|c|c|c|c|}
\hline History & Examination & Treatment & Prognosis \\
\hline $\begin{array}{l}\text { Factors increasing index of } \\
\text { suspicion of RCRSP: } \\
\text { Pain over the deltoid/ upper } \\
\text { arm } \\
\text { Activity related } \\
\text { Minimal pain at rest except } \\
\text { when lying on affected side } \\
\text { Associated with changes in } \\
\text { load from a specific activity or } \\
\text { repetitive use. Consider } \\
\text { changes in occupation or } \\
\text { participation demands. } \\
\text { Factors reducing index of } \\
\text { suspicion of RCRSP: } \\
\text { Complaint of subluxation or } \\
\text { dislocation } \\
\text { Pain in the neck } \\
\text { Distal neurovascular symptoms }\end{array}$ & $\begin{array}{l}\text { Factors increasing index of } \\
\text { suspicion of RCRSP: } \\
\text { Familiar pain is reproduced } \\
\text { with resisted testing of the } \\
\text { shoulder, usually abduction } \\
\text { and lateral rotation } \\
\text { Imaging not recommended as } \\
\text { a first line investigation unless } \\
\text { red flag pathology suspected, } \\
\text { for example tumour or } \\
\text { fracture }\end{array}$ & 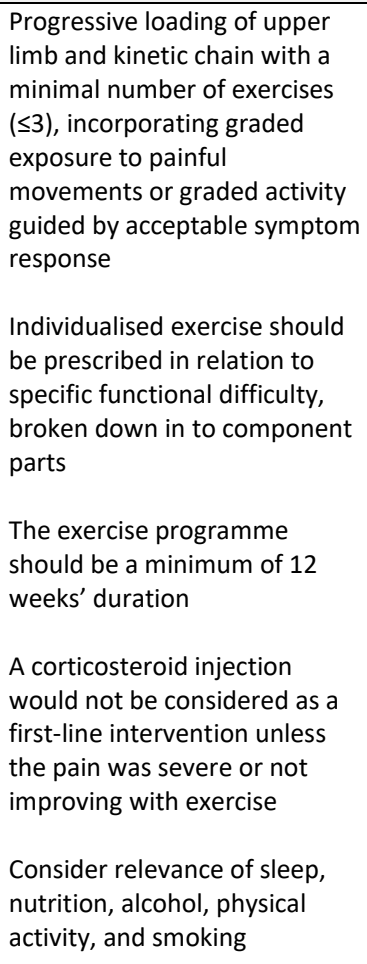 & $\begin{array}{l}\text { Prognosis is likely to be } \\
\text { favourable but this will take a } \\
\text { minimum of } 12 \text { weeks, but } \\
\text { further improvement may be } \\
\text { expected up to } 24 \text { weeks } \\
\text { Referral for investigation or } \\
\text { orthopaedic opinion would be } \\
\text { considered for patients whose } \\
\text { symptoms remain } \\
\text { unacceptable despite an } \\
\text { appropriate period of } \\
\text { engagement with an exercise } \\
\text { based management approach } \\
\text { or if the patient remains } \\
\text { anxious or unconvinced about } \\
\text { the treatment approach. }\end{array}$ \\
\hline
\end{tabular}

Table 2 Summary of recommendations

What key information is required from the history to raise the index of suspicion of this classification or diagnosis over other shoulder pain presentations?

The majority opinion of the group was that pain should be broadly over the deltoid and upper arm region to raise the suspicion of this sub-group of shoulder pain. If there is complaint of subluxation or dislocation as the primary problem, then the index of suspicion would be reduced. For this sub-group of shoulder pain, pain is activity related (better or worse), typically worse reaching overhead or behind the back, with minimal pain at rest except when lying on the affected shoulder. Pain in the neck, reproduction of shoulder pain on movement of the neck, and distal neurovascular symptoms would reduce the index of suspicion. 
Do you consider age, gender and occupation as relevant factors within your diagnostic reasoning with regard to 'rotator cuff syndrome'? If so, why and how?

The majority opinion of the group was that changes in occupation or participation demands rather than occupation alone were relevant in raising the index of suspicion of RCRSP. Majority opinion on other factors was not reached.

What key information is required from the physical examination to confirm this classification or diagnosis? The majority opinion of the group was that there should be no significant loss ( $>50 \%$ ) of passive range of movement in any direction, particularly external rotation. Typically, familiar pain should be reproduced with loading/ resisted testing, usually abduction and lateral rotation. In keeping with findings from the history to raise the index of this sub-group of shoulder pain, movement of the neck should not produce or abolish the shoulder pain and there should be no neurological signs, including upper limb mechano-sensitivity tests.

What is the role of imaging in confirming this classification or diagnosis?

The majority opinion of the group was that imaging is useful to rule out red flag pathology if the index of suspicion is raised during the history and physical examination, e.g. tumour or fracture or if suspected dislocation. In the absence of stiffness and with a consistent history, as described above, the group consensus was that imaging would not be indicated initially due to the poor correlation between pain and structural pathology. Imaging findings do not influence non-surgical management options and could potentially be a barrier to patient engagement, but imaging may have a role if patients do not follow the expected trajectory of improvement.

What is the preferred terminology for this shoulder pain classification?

The majority opinion of the group was that RCRSP or weak and painful shoulder were the preferred descriptors but this depends on context, including who we are communicating with and what the patient has been told before. The majority opinion of the group was that 
healthcare professionals, including GPs, orthopaedic surgeons, radiologists, physiotherapist, should now avoid using the term 'impingement' but it is clear that discourse is still needed regarding the most useful term(s).

In summary, what is 'rotator cuff syndrome'?

The majority opinion of the group conceptualised RCRSP as symptoms of pain and signs of weakness related to the rotator cuff when loaded, and movement related shoulder pain without significant stiffness.

How do you explain 'rotator cuff syndrome' to your patients?

The majority opinion of the group was that this could be explained as 'an issue with the muscles and tendons of the shoulder, they're lacking strength, capacity, tolerance and fitness - that's why they complain when you lift your arm.'

What is/ are the preferred methods of treatment?

The majority opinion of the group was that loading through exercise, including progressions and regressions, within acceptable symptom response, was the preferred approach to treatment. Progressive loading of upper limb and kinetic chain was advocated by the majority incorporating graded exposure to painful movements or graded activity. Cognitive behavioural principles may need to be adopted if there is evidence of avoidance behaviour.

A steroid injection would not be considered as a first-line intervention by the majority unless the pain was severe or not improving with exercise over an acceptable period of time (e.g. 6 to 12 weeks). Beyond a programme of physiotherapist-led exercise, further referral for investigation or orthopaedic opinion would be considered for patients whose symptoms remain unacceptable despite an appropriate period of engagement with an exercise based 
management approach or if the patient remains anxious or unconvinced about the treatment approach.

Do you consider the role of lifestyle, e.g. sedentary behaviour, and metabolic factors, e.g. obesity when assessing and treating patients with this pain presentation?

The majority opinion of the group was that assessment of sleep, nutrition, alcohol, physical activity, and smoking should be conducted with promotion of change where relevant to the patient. However, how to recognise, implement and support behaviour change remains unclear but the majority opinion of the group was that brief interventions should be incorporated to make every contact count with regard to lifestyle and musculoskeletal health.

If exercise is prescribed, what are the preferred parameters guiding this prescription?

Type of exercise and the factors that inform this

The majority opinion of the group was to use isometric, isotonic or eccentric exercise according to what is most acceptable to the patient while still providing sufficient stimulus to challenge what is weak and work to fatigue. The majority opinion was to prescribe exercise to challenge specific functional difficulty but break down into component parts within limits of acceptable symptom response and to stage loading according to patient's ability and symptom irritability. Majority opinion on more specific parameters was not reached.

Number of sets and repetitions and the factors that inform this The majority opinion of the group was that this should be individualised based on acceptable symptom response and functional requirement, e.g. speed vs sustained. Majority opinion on more specific parameters was not reached. 
Frequency of exercise and the factors that inform this

The majority opinion of the group was that if patients are exercised to the point of muscle fatigue, exercise on alternate days. If heavy load or plyometric exercises are included, this should be limited to exercise over two to three sessions per week. Majority opinion on more specific parameters was not reached.

Number of exercises and the factors that inform this

The majority opinion of the group was that a minimal number of different exercises, rarely more than three, should be prescribed. Exercises should be adapted to address the specific functional difficulties that the patient reports and be developed in the context of their physical capabilities, functional deficits and available time to devote to exercise.

Intensity/ effort of exercise and the factors that inform this

The majority opinion of the group was that this should be adapted according to patient specific acceptable symptom response.

Painful versus painless and the factors that inform this

The majority opinion of the group was that exercise could provoke pain providing that it settles sufficiently to enable the patient to perform the next set of exercises shortly afterwards within a session. However, pain provocation was not seen as an essential feature of the exercise as long as the patient is being challenged by the exercise.

Duration of exercise

The majority opinion of the group was that an exercise programme should be adhered to over a minimum of 12 weeks, but sometimes a longer period, up to 24 weeks, will be required.

Which factors or characteristics do you see as important indicators of prognosis?

The majority opinion of the group was that a number of factors might be relevant including patients' attitudes regarding pain and exercise, fear avoidance, litigation, lifestyle factors, 
e.g. smoking, sedentary behaviour, diet, alcohol, educational levels, multiple pain sites, level of baseline pain and disability, previous experience and response to treatment, patient expectations regarding recovery and physiotherapy, co-morbidities, levels of self-efficacy, psychological distress, and social circumstances, e.g. employment, social engagement and hobbies.

What is the expected prognosis of 'rotator cuff syndrome'?

The majority opinion of the group was that patients, and other clinical colleagues, should be advised that the prognosis is likely to be favourable but this will take a minimum of 12 weeks, but further improvement may be expected up to 24 weeks.

\section{Discussion}

This consensus exercise has resulted in suggestions regarding patient history and physical examination findings that might raise the index of suspicion of RCRSP as well as suggestions regarding prognosis and suggested terminology to describe this common presentation to patients and clinical colleagues. Further suggestions related to non-surgical management are reported as well as factors that might influence the response to this. In summary, the suggestions for non-surgical management include a minimal number of exercises prescribed to challenge the functional deficit of the patient over a minimum 12-week period. Unless there is concern about red flag pathology then imaging was not regarded as useful unless the patient did not respond as expected. Similarly, steroid injections would not be considered as a first-line intervention unless pain was severe and preventing engagement with exercise.

The caution regarding use of imaging expressed through this consensus approach is unsurprising given the now well recognised lack of association between such findings, 
structural diagnosis and the pain that patients complain of $(4,16)$. Similar caution with regards to use of steroid injection is also not surprising given contemporary research evidence highlighting concerns about this treatment approach for patients with RCRSP $(17,18)$.

The dearth of evidence to inform the specific prescription parameters of an exercise programme for RCRSP has been recognised (9). The findings from this consensus exercise are broadly in agreement with the scant research evidence-based guidance and there is overlap with the previous consensus exercise undertaken by physiotherapists in 2012 (14) in terms of history, physical examination, use of imaging and exercise as the mainstay of treatment.

There is some divergence between the consensus reports with regards to the specifics of exercise prescription though with, arguably, a more progressive approach suggested via this current consensus exercise. There is also divergence with regards to expected response time and prognosis, with this current consensus exercise suggesting a minimum of 12-weeks required, often longer, depending on patient factors including baseline levels of pain and disability, expectations of physiotherapy, social circumstances etc. Also within this current consensus exercise there was recognition of the potential for lifestyle factors to influence the onset and persistence of RCRSP. Identification of such lifestyle factors, including smoking and physical activity, were seen as important although the optimal way to address these factors within a physiotherapy setting was less clear. 
One clear finding from this current consensus exercise is the lack of agreement on the specific exercise prescription parameters including type and dose of exercise. Given the lack of research evidence this is perhaps unsurprising but given the widespread recognition now that exercise should be the mainstay of treatment for RCRSP this is a clear gap that needs to be addressed through well-designed, adequately powered, randomised controlled trials. However, it should be recognised that RCRSP affects a diverse and heterogenous population and therefore it is questionable whether a homogeneous type or dosage of exercise will be found. Evaluations that are required include comparison of different types of exercise, e.g. standardised versus individualised, painful versus non-painful, while controlling for other parameters that might influence outcome such as dose of exercise (sets and repetitions). Furthermore, evaluation of the comparative effectiveness of different doses should ensure that other factors including the type of exercise, are adequately controlled to enable a valid comparison. Once a guiding framework, rather than a prescriptive recipe, for the optimal prescription parameters has been established then it would be appropriate to evaluate this against other approaches, including 'wait-and-see', to establish whether any effects of exercise, and other approaches, are due to reasons other than natural history and placebo.

Further evaluation could focus on approaches to optimise exercise adherence, approaches to identify and address relevant psychosocial factors, evaluation of the impact of lifestyle factors and whether important diagnostic and prognostic factors upon which to stratify care for RCRSP can be identified.

\section{Implications}

This report is by no means definitive in its recommendations but provides an opportunity for clinicians and researchers to reflect on their own current practice and consider the 
differences between the approaches described in this paper and their own approach. This process offers the potential to consider such questions as; 'why do we do what we do?' and 'why do we believe what we believe?' Hence, the paper provides a benchmark for reflection and a stimulus to challenge practice while at the same time highlighting the many areas of uncertainty that still exist in this area and require further research.

\section{Limitations}

This is a report of a consensus developed through consultation with physiotherapists who have clinical and academic expertise in shoulder pain. Such processes are necessarily reflective of the individuals involved and are not necessarily generalisable. Furthermore, consensus was based on achieving a majority through a voting system based on agreement or not. When aiming to synthesise complex processes into a digestible report, such compromises are necessary.

\section{Conclusion}

The findings from this consensus exercise suggest clear features in the history and physical examination that raise the index of suspicion of RCRSP. Furthermore, suggestions have been made about preferred terminology and factors that might impact on prognosis. In the absence of suspicion of red flag pathology, caution regarding the use of imaging was suggested because the findings do not inform initial treatment choices and could be nocebic. Exercise is recognised as the mainstay of treatment for RCRSP with agreement that a minimal number of exercises should be prescribed to challenge the functional deficit of the patient over a minimum of 12 -week period. However, there was little agreement beyond this. This is reflective of the dearth of research evidence available to inform optimal exercise prescription for RCRSP. Given the widespread recognition now that exercise should 
be the mainstay of treatment for RCRSP this is a clear gap that needs to be addressed through well-designed, adequately powered, randomised controlled trials. 
1. Lewis J. Rotator cuff related shoulder pain: Assessment, management and uncertainties. Man Ther. Elsevier Ltd; 2016;23:57-68.

2. Littlewood C, May S, Walters S. Epidemiology of rotator cuff tendinopathy: a systematic review. Shoulder Elb. 2013;5(4):256-65.

3. Cuff A, Littlewood C. Subacromial impingement syndrome - What does this mean to and for the patient? A qualitative study. Musculoskelet Sci Pract. 2018;33:24-8.

4. Chester R, Jerosch-herold C, Lewis J, Shepstone L. Psychological factors are associated with the outcome of physiotherapy for people with shoulder pain : a multicentre longitudinal cohort study. Br J Sports Med. 2018;52:269-75.

5. Dean B, Gwilym S, Carr A. Why does my shoulder hurt? A review of the neuroanatomical and biochemical basis of shoulder pain. Br J Sports Med. 2013;47(17):1095-104.

6. Bury J, Littlewood C. Rotator cuff disorders: a survey of current (2016) UK physiotherapy practice. Shoulder Elb. 2018;10(1):52-61.

7. Ketola S, Lehtinen J, Arnala I, Nissinen M, Westenius H, Sintonen $\mathrm{H}$, et al. Does arthroscopic acromioplasty provide any additional value in the treatment of shoulder impingement syndrome? A two year randomised controlled trial. J Bone Jt Surg. 2009;91:1326-34.

8. Littlewood C, May S, Walters S. A review of systematic reviews of the effectiveness of conservative interventions for rotator cuff tendinopathy. Shoulder Elb. 2013 Mar 4;5(3):15167.

9. Littlewood C, Malliaras P, Chance-Larsen K. Therapeutic exercise for rotator cuff tendinopathy: a systematic review of contextual factors and prescription parameters. Int J Rehabil Res [Internet]. 2015;38(2):95-106.

10. Smith BE, Hendrick P, Smith TO, Bateman M, Moffatt F, Rathleff MS, et al. Should exercises be painful in the management of chronic musculoskeletal pain? A systematic review and metaanalysis. Br J Sports Med. 2017;1-10.

11. Beard DJ, Rees JL, Cook JA, Rombach I, Cooper C, Merritt N, et al. Arthroscopic subacromial decompression for subacromial shoulder pain (CSAW): a multicentre, pragmatic, parallel group, placebo-controlled, three-group, randomised surgical trial. Lancet. 2017;6736(17):110.

12. Paavola M, Malmivaara A, Taimela S, Kanto K, Inkinen J, Kalske J, et al. Subacromial decompression versus diagnostic arthroscopy for shoulder impingement : randomised, placebo surgery controlled clinical trial. BMJ. 2018;362:k2860.

13. Littlewood C, Lowe A, Moore J. Rotator cuff disorders: Survey of current UK physiotherapy practice. Shoulder Elb. 2012;4:64-71.

14. Klintberg IH, Cools AMJ, Holmgren TM, Moser JS, Spunton V, Ginn K. Consensus for physiotherapy for shoulder pain. Int Orthop. 2015;39(4):715-20.

15. Griffin D, Dickenson E, Donnell J, Agricola R, Awan T, Beck M, et al. The Warwick Agreement on femoroacetabular impingement syndrome ( FAl syndrome ): an international consensus statement. Br J Sport Med. 2016;50:1169-76.

16. Frost $P$, Andersen J, Lundorf $E$. Is supraspinatus pathology as defined by magnetic resonance impaging associated with clinical signs of shoulder impingement? J Shoulder Elb Surg. 1999;8:565-8.

17. Mohamadi A, Chan JJ, Claessen FMAP, Ring D, Chen NC. Corticosteroid Injections Give Small and Transient Pain Relief in Rotator Cuff Tendinosis: A Meta-analysis. Clin Orthop Relat Res. Springer US; 2017;475(1):232-43.

18. Dean BJF, Franklin SL, Murphy RJ, Javaid MK, Carr AJ. Glucocorticoids induce specific ionchannel-mediated toxicity in human rotator cuff tendon: a mechanism underpinning the ultimately deleterious effect of steroid injection in tendinopathy? Br J Sports Med. 2014;48(22):1620-6. 
